\title{
Cryptococcal meningitis - a silent culprit behind delirium in a patient with Parkinson's disease, on levodopa, presenting with urinary tract infection
}

\author{
Robin Manappallil \\ Department of Internal Medicine, National Hospital, Calicut, Kerala, India
}

\begin{abstract}
Patients with Parkinson's disease are prone to mental disturbances like psychosis and delirium. Psychosis has been attributed to drugs like dopamine agonists, while delirium is commonly seen during the days of hospital stay and infections. This is a case of a 62-year-old lady, a known case of Parkinson's disease on levodopa therapy, who has had repeated episodes of delirium over the past year. Her delirium episodes were usually associated with urinary tract infection and hyponatremia or as a part of levodopa therapy. However, this time on further evaluation, she was found to have cryptococcal meningitis as the culprit for her delirium.
\end{abstract}

\section{Introduction}

Parkinson's disease (PD) mostly affects the elderly age group. Delirium is an acute organic brain syndrome characterized by cognitive impairment, attention deficits, decreased level of consciousness, varied psychomotor activity, and wake - sleep rhythm disorders. ${ }^{1}$ It has been reported that the incidence of delirium increases with age, hospital stay and as a part of levodopa therapy. ${ }^{2,3}$ Urinary tract infections (UTI) may be a cause for delirium in elderly population., ${ }^{4,5}$ Confusional psychosis has been reported in cryptococcal meningitis. ${ }^{6}$

This case report aims at highlighting the need to rule out meningitis in a patient with PD on levodopa therapy presenting in delirium.

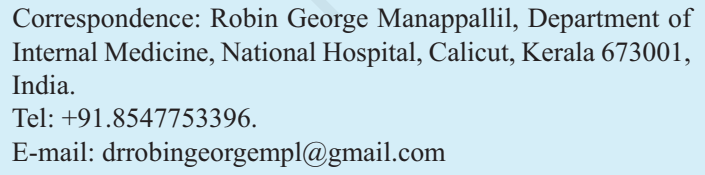

Key words: Delirium; Parkinson's disease; levodopa; urinary tract infection; meningitis.

Received for publication: 5 July 2015.

Revision received: 23 September 2015.

Accepted for publication: 1 October 2015.

This work is licensed under a Creative Commons Attribution NonCommercial 4.0 License (CC BY-NC 4.0).

CCopyright R. Manappallil, 2016

Licensee PAGEPress, Italy

Italian Journal of Medicine 2016; 10:154-156

doi:10.4081/itjm.2016.619

\section{Case Report}

This is a case of a 62-year-old female who was brought with history of delirium for the past week. She did not have any symptoms of fever, headache or vomiting. There were no other systemic complaints. The patient is a diabetic with diabetic nephropathy and is on insulin therapy. She is also a known case of PD on levodopa $100 \mathrm{mg}+$ carbidopa $10 \mathrm{mg}$ three times a day for the past 8 years. She has been experiencing such delirium episodes over the last year, for which the cause was either hyponatremia following UTI or levodopa induced. The patient used to improve when her hyponatremia and UTI got corrected or when levodopa was withheld for a few days.

On examination, patient was conscious but mildly disoriented. She was afebrile with heart rate of 70 beats/min, blood pressure of 140/90 $\mathrm{mmHg}$ and respiratory rate of 24 breaths/min with saturation $94 \%$ in room air. Neurological examination revealed bradykinesia, upper limb tremors and cogwheel rigidity. She had minimal neck stiffness, which was thought to be because of PD and her uncooperative nature. Kernig's and Brudzinski's signs were negative and ophthalmic examination was normal. Her other systemic examinations were normal.

Her investigation reports revealed a normal hemogram, deranged renal functions (urea $50 \mathrm{mg} / \mathrm{dL}$ and creatinine $2.5 \mathrm{mg} / \mathrm{dL}$ ) and normal electrolytes and liver function tests. Her chest X-ray and electrocardiogram were normal and computed tomography of the brain showed age related atrophic changes. Her viral markers like HBsAg, anti HBs, anti-hepatitis C virus and HIV were negative. Thyroid profile and anti thyroid peroxidase antibody were normal. Her urine microscopy showed presence of plenty of pus cells 
and culture grew Escherichia coli. Based on the history, examination and investigation findings, a provisional diagnosis of sepsis due to UTI and delirium secondary to either levodopa therapy or complicated by UTI in elderly with PD was considered.

She was started on intravenous meropenum as per culture sensitivity report, in renal dosage. Levodopa was withheld and quetiapine was started, as advised by the psychiatrist. However, after 4 days of treatment, there was no improvement in her delirium. Her repeat urine culture was sterile. On day 5 , cerebrospinal fluid (CSF) analysis was done, which was normal in pressure with mild lymphocytic pleocytosis, mildly decreased sugars but normal proteins. Yeast cells were visualized and cryptococcal meningitis was confirmed with India ink and culture. Cryptococcal antigen was also positive in blood.

The patient's relatives were informed about the diagnosis and use of amphotericin B for treatment along with its nephrotoxic property. In the setting of diabetic nephropathy, relatives were not willing for amphotericin $\mathrm{B}$. The patient relatives belonged to a very low socioeconomic status and were not able to afford liposomal AmB. Moreover, since this was the first case of cryptococcal meningitis in our hospital, the availability of liposomal AmB and flucytosine (which forms the induction phase therapy) was another issue. As a result, she was started on low dose fluconazole and her renal functions were monitored. Over the next few days, the patient started showing improvement with regard to her delirious state and by day 12 , she was well oriented. She was discharged on day 15 and was asked to continue fluconazole for other 6 weeks, with weekly review of her renal functions. Levodopa was restarted in lower doses.

\section{Discussion}

PD is a neurodegenerative disorder characterized by bradykinesia, rest tremor, muscular rigidity, shuffling gait, and flexed posture. It can be accompanied by autonomic, sensory, sleep, cognitive, and psychiatric disturbances. Parkinsonism results from a reduction in dopaminergic transmission within the basal ganglia. Although the vast majority of cases appear to be sporadic, there is increasing evidence that genetic factors play an important role in many forms of PD. PARK1, PARK4, and PARK5 lead to an autosomal dominant form of PD while PARK2 and PARK7 lead to autosomal recessive disorders. Pathologically, there is mild frontal atrophy with loss of the normal dark melanin pigment of the midbrain and microscopically there is a degeneration of the dopaminergic cells with the presence of Lewy bodies in the remaining neurons and processes of the substantia nigra pars compacta. Lewy bodies have a high concentration of $\alpha$-synuclein and its mutation can cause familial PD. Levodopa remains the most effective treatment for PD as it significantly improves motor symptoms and increases quality of life. ${ }^{7}$

Delirium is a syndrome marked by over activity, sleeplessness, tremulousness and vivid hallucinations. It may be associated with convulsions. The common causes of delirium include septicemia, thyrotoxicosis, neoplasms, head injury, subarachnoid hemorrhage, meningitis, alcohol withdrawal and drug intoxications. ${ }^{8}$ The pathophysiology of delirium revolves around the mesocortical dopaminergic system. ${ }^{9}$ The occurrence of delirium in Parkinson patients has been attributed to the treatment with levodopa. Confusion and psychosis are seen in advanced cases of $\mathrm{PD}$, where the patients have been taking high doses of levodopa for many years. Other behavioural alterations encountered with levodopa include dopamine dysregulation syndrome and punding. ${ }^{7}$ Studies have shown that elderly population is prone for delirium, especially during hospital stay and in the setting of UTI. ${ }^{2-5,10}$

Meningitis is an infection of the subarachnoid space. It may be bacterial, viral, fungal or rickettsial. Fever, headache and stiff neck form the classical triad of meningitis. A decreased level of consciousness, nausea, vomiting, photophobia and seizures may also occur. Cryptococcal meningitis has been reported to cause confusional psychosis. ${ }^{6}$ Other meningeal signs include Kernig and Brudzinski. CSF analysis forms the mainstay for the diagnosis of meningitis.

In this case, the patient is suffering from PD and is on levodopa therapy for the same. Hence, her previous episodes of delirium where thought to be levodopa induced, as she had no other symptoms. Levodopa used to be withheld for a few days and then restarted in lower doses once she was asymptomatic. She also had UTI during some of her delirium episodes. During her present admission for delirium, she had UTI, but her symptoms persisted in spite of UTI treatment and stopping of levodopa. This raised the need for CSF analysis, which showed cryptococcal meningitis as the cause for her delirium. The yeast like fungus Cryptococcus makes use of levodopa as substrate to produce melanin which appear to be related to the virulence of the strain. In particular melanin gives resistance against host defence. The symptoms were thought to be levodopa induced and remissions obtained when levodopa was withheld for few days. Possibly the yeast became less virulent without this substrate.

\section{Conclusions}

Patients with PD are prone for delirium. The causes can be due to levodopa or infections like UTI, which can also induce hyponatremia. Old age can also 
contribute to delirium. This case report highlights the importance of considering meningitis, even in the absence of meningeal signs and symptoms, in a patient with PD presenting in delirium, who continues to be symptomatic in spite stopping of levodopa and treating infections like UTI.

\section{References}

1. Lipowski ZJ. Update on delirium. Psychiatric Clin N Am 1992;15:335-46.

2. Noé-Sebastián E, Irimia-Sieira P, Pomares-Arias E, et al. Neuropsychiatric disorders in Parkinson's disease [Spanish]. Rev Neurol 2001;32:676-81.

3. Inouye $\mathrm{SK}$, van Dyck $\mathrm{CH}$, Alessi CA, et al. Clarifying confusion: the confusion assessment method. A new method for detection of delirium. Ann Inter Med 1990;113:941-8.

4. Balogun SA, Philbrick JT. Delirium, a symptom of UTI in the elderly: fact or fable? a systematic review. Can Geriatr J 2014;17:22-6.
5. Eriksson I, Gustafson Y, Fagerström L, Olofsson B. Urinary tract infection in very old women is associated with delirium. Int Psychogeriatr 2011;23:496-502.

6. Sa'adah MA, Araj GF, Diab SM, Nazzal M. Cryptococcal meningitis and confusional psychosis. A case report and literature review. Trop Geogr Med 1995;47:224-6.

7. Mahon RD, Jorge LJ. Parkinson's disease and other extrapyramidal movement disorders. In: Fauci AS, Braunwald E, Kasper DL, Hauser SL, Longo DL, Jameson JL, Loscalzo J, eds. Harrison's principles of internal medicine. 17th ed. Vol. 2. New Delhi: McGraw Hill Education; 2008. pp 2549-2559.

8. Ropper AH, Samuels MA. Delirium and other acute confusional states. In: Ropper AH, Samuels MA, eds. Adams and Victor's principles of neurology. 9th ed. New Delhi: McGraw Hill Education; 2009. pp 405-406.

9. Rummans TA, Evans JM, Krahn LE, Fleming K. Delirium in elderly patients: evaluation and management. Mayo Clin Proc 1995;70:989-98.

10. Manepalli J, Grossberg GT, Mueller C. Prevalence of delirium and urinary tract infection in a psychogeriatric unit. J Geriatr Psychiatry Neurol 1990;3:198-202. 